\title{
Correction to: Economic efficiency of rainfed wheat farmers under changing climate: evidence from Pakistan
}

\section{Nasir Mahmood ${ }^{1,2,3} \cdot$ Muhammad Arshad $^{1,3} \cdot$ Harald Kächele $^{1,4} \cdot$ Ayat Ullah $^{1} \cdot$ Klaus Müller $^{1,3}$}

Published online: 3 July 2020

(C) Springer-Verlag GmbH Germany, part of Springer Nature 2020

\section{Correction to: Environmental Science and Pollution Research} https://doi.org/10.1007/s11356-020-09673-5

The correct Eq. 4 is presented in this paper.

The original article is corrected.

$$
\begin{aligned}
\ln y_{i}= & \beta_{o}+\beta_{1} \ln x_{1}+\beta_{2} \ln x_{2}+\beta_{3} \ln x_{3}+\beta_{4} \ln x_{4} \\
& +\beta_{5} \ln x_{5}+\beta_{6} \ln x_{6}+1 / 2 \beta_{7} \ln x_{1}^{2}+1 / 2 \beta_{8} \ln x_{2}^{2} \\
& +1 / 2 \beta_{9} \ln x_{3}^{2}+1 / 2 \beta_{10} \ln x_{4}^{2}+1 / 2 \beta_{11} \ln x_{5}^{2} \\
& +1 / 2 \beta_{12} \ln x_{6}^{2}+\beta_{13} \ln x_{1} \ln x_{2}+\beta_{14} \ln x_{1} \ln x_{3} \\
& +\beta_{15} \ln x_{1} \ln x_{4}+\beta_{16} \ln x_{1} \ln x_{5}+\beta_{17} \ln x_{1} \ln x_{6} \\
& +\beta_{18} \ln x_{2} \ln x_{3}+\beta_{19} \ln x_{2} \ln x_{4}+\beta_{20} \ln x_{2} \ln x_{5} \\
& +\beta_{21} \ln x_{2} \ln x_{6}+\beta_{22} \ln x_{3} \ln x_{4}+\beta_{23} \ln x_{3} \ln x_{5} \\
& +\beta_{24} \ln x_{3} \ln x_{6}+\beta_{25} \ln x_{4} \ln x_{5}+\beta_{26} \ln x_{4} \ln x_{6} \\
& +\beta_{27} \ln x_{5} \ln x_{6}+\left(v_{i}-u_{i}\right)
\end{aligned}
$$

The online version of the original article can be found at https://doi.org/ $10.1007 / \mathrm{s} 11356-020-09673-5$

Publisher's note Springer Nature remains neutral with regard to jurisdictional claims in published maps and institutional affiliations.

Nasir Mahmood

nasir.mahmood@zalf.de

1 Leibniz Centre for Agricultural Landscape Research (ZALF),

Eberswalder Straße 84, 15374 Müncheberg, Germany

2 Department of Economics \& Agricultural Economics, PMAS-Arid Agriculture University, Rawalpindi, Pakistan

3 Albrecht Daniel Thaer Institute of Agricultural and Horticultural Sciences, Humboldt University, 10117 Berlin, Germany

4 Eberswalde University for SustainableDevelopment, Schicklerstraße 5, 16225 Eberswalde, Germany 